Original Paper http://ajol.info/index.php/ijbcs http://indexmedicus.afro.who.int

\title{
Effets des changements climatiques et des modes de gestion sur la fertilité des sols dans la commune de Banikoara au nord-ouest du Benin
}

\author{
S. KATE ${ }^{1}$, A. H. AZONTONDE ${ }^{2 *}$, G. D. DAGBENONBAKIN ${ }^{1}$ et B. SINSIN $^{3}$ \\ ${ }^{1}$ Institut National des Recherches Agricoles du Bénin, BP 884, Agonkanmey, \\ Abomey-Calavi 213500 70, Bénin. \\ ${ }^{2}$ Laboratoire des Sciences du Sol, Eaux et Environnement, BP 988, Agonkanmey, Abomey-Calavi. \\ ${ }^{3}$ Laboratoire d'Ecologie Appliquée, FSA, UAC 01 BP 526, Tel/Fax (229) 21-30-30-84, Cotonou, Bénin. \\ *Auteur correspondant; E-mail : anastase12000@yahoo.fr; Tel: (229) 97762165
}

\section{RESUME}

Le présent diagnostic réalisé dans la commune de Banikoara vise à étudier les effets des changements climatiques et des modes de gestion sur la fertilité des sols. A cet effet, les caractéristiques morphologiques et physico-chimiques de 6 unités de sols répétées 3 fois dans 3 différents arrondissements ont été déterminées et comparer à ces mêmes caractéristiques déterminées en 1971. Les résultats d'analyses physicochimiques ont été soumis à des analyses statistiques. Il ressort de l'étude des 2 séries de caractéristiques constituées de ces résultats que les taux de matière organique des différentes unités pédologiques de la Commune de Banikoara ont varié très significativement entre 1971 et $2010(p<0,01)$. Cependant, ces taux de matière organique n'ont pas varié significativement suivant les unités pédologiques ( $p>0,05$ ). Il en est de même pour les taux d'azote des différentes unités pédologiques qui ont connu une variation très significative entre la période de 1970 et 2013 ( p <0,01) mais n'ont pas varié significativement d'une unité à l'autre dans la même série. Les résultats d'analyse de la variance indiquent que les teneurs des bases échangeables et de la capacité d'échange cationique ont varié significativement $(\mathrm{p}<0,01)$ entre 1971 et 2010 et suivant les unités pédologiques $(\mathrm{p}<0,01)$. Les changements climatiques ont donc eu un impact négatif très significatif sur la fertilité des sols pendant les 40 années d'exploitation, alors que les modes de gestion des sols n'ont induit entre eux aucune différence significative au niveau de la matière organique et de l'azote, paramètres majeurs de la fertilité des sols. Par ailleurs, les niveaux de fertilité des unités pédologiques sont passés de moyen en 1971 à très bas en 2010 en dehors des sols hydromorphes et des sols ferrugineux hydromorphes sur roche basique dont les niveaux de fertilité sont restés moyens et moyen à bas respectivement.

(c) 2016 International Formulae Group. All rights reserved.

Mots clés : Changements climatiques, niveau de fertilité, carbone, azote, hydromorphe, ferrugineux.

\section{Impact of climate change and types of soil management on soil fertility in Banikoara municipality at the North West of Benin}

\begin{abstract}
The soil survey conducted throughout the Banikoara municipality within the diagnostic effects of climate change on soil fertility was used to determine the morphological and physico-chemical characteristics
\end{abstract}


of six soil units repeated three times in three different districts and compare them to those same characteristics determined in 1971, 40 years earlier. It appears from the study of two sets of characteristics through statistical analyzes made from these results that the rate of carbon or organic matter in different soil units of the municipality of Banikoara has varied significantly between 1970 and $2010(p<0.01)$. However, the carbon content did not vary significantly between the soil units $(p>0.05)$. It is the same for the different soil nitrogen units that have experienced a very significant variation between the period 1971 and 2010 rates $(\mathrm{p}<0.01)$ but did not differ significantly between one unit to another in the same series. The ANOVA results indicate that the levels of exchangeable bases (BE) and cation exchange capacity (CEC) varied significantly ( $p<0.01$ ) between 1971 and 2010 and according to the soil units $(\mathrm{p}<0.01)$. Climate change has thus had a significant negative impact on soil fertility during the 40 years of operation, while the modes of land management did not induce a significant difference between them in terms of organic matter and nitrogen major parameters of the soil fertility. Furthermore, levels of fertility soil units went from average in 1971 to very low in 2010 out of waterlogged soils and waterlogged ferruginous soils on basic rock whose fertility levels have remained low medium and medium respectively.

(C) 2016 International Formulae Group. All rights reserved.

Keywords: Climate change, soil fertility, carbon, nitrogen, hydromorphic, ferrugenous.

\section{INTRODUCTION}

Les questions des changements climatiques préoccupent les scientifiques et les décideurs politiques en raison de leurs conséquences immédiates et durables sur l'environnement (Issa, 1995; IPCC, 1996 ; Ogouwalé, 2006). Les analyses faites sur l'évolution par GIEC (2007) ont prouvé une modification de l'équilibre énergétique du système «Terre-Océan-AtmosphèreBiosphère». Un climat modifié qui se traduirait par une amplification des phénomènes extrêmes dans certains pays des régions intertropicales aura des impacts sur l'agriculture et par conséquent sur la sécurité alimentaire. Ainsi, selon Conway et Toenniessen (2003), les terres cultivables, les pâturages et les forêts qui occupent $60 \%$ de la superficie de la terre sont progressivement exposés aux menaces de la variabilité climatique croissante et par la suite des changements climatiques. Par ailleurs GIEC (2007) affirme que l'Afrique de l'Ouest est particulièrement vulnérable à la variabilité et aux changements climatiques notamment à cause de certaines de ses caractéristiques physiques et socio-économiques qui la prédisposent à être affectée de façon disproportionnée, par les effets négatifs des variations du climat. Les projections du GIEC (2007) établissent que vers l'an 2020, la production agricole et l'accès à la nourriture seront sérieusement compromis par la variabilité et l'évolution du climat. Ceci est d'autant plus vrai que l'agriculture est sans conteste, le secteur le plus important dans les économies de la plupart des pays africains non producteurs du pétrole; elle participe, selon la FAO (2007), pour près de 20 à $30 \%$ au PIB et $55 \%$ aux revenus d'exportation et $70 \%$ de la population du continent en dépendent pour leur survie.

L'agriculture africaine reste essentiellement pluviale et de subsistance, en dépit d'un potentiel énorme en matière d'irrigation, ce qui la rend assez vulnérable aux modifications du climat.

Les modèles climatiques régionaux prédisent en effet que la combinaison des facteurs que sont la hausse des températures, la baisse et l'irrégularité des pluies, la montée des eaux de mer, les situations extrêmes (inondations, sécheresses, etc.) résultent inéluctablement en une réduction sensible de la production et de la productivité agricoles, une plus grande sensibilité des cultures aux attaques des ravageurs, une réduction des ressources en eau disponibles, une baisse de la fertilité des sols et de la productivité animale. Aussi, un impact considérable se fera-t-il ressentir sur la disponibilité des ressources humaines pour le secteur agricole, le nouvel environnement climatique créant les conditions favorables au développement de 
maladies déjà endémiques (paludisme, maladie de sommeil, méningite, etc.). Pour l'OMM et PNUE (2002), pour réduire les effets néfastes, directs ou indirects potentiels des changements climatiques sur le système agroalimentaire, les populations doivent s'adapter et les systèmes économiques devront être adaptés aux futurs contextes climatiques.

$\mathrm{Au}$ Bénin où l'agriculture constitue la base de l'économie avec une contribution de $36 \%$ au PIB (SRPA, 2008) et de $88 \%$ aux recettes d'exportation, les impacts négatifs du changement climatique ne sont plus à démontrer. Le secteur agricole, pourvoyeur de ressources alimentaires et financières, est très affecté et mérite de ce fait une attention particulière si le Bénin ambitionne de s'assurer une autosuffisance alimentaire. La projection de la situation future du climat à travers les différents scénarii n'est guère en faveur d'une quelconque prospérité agricole car il est prévu un changement des paramètres climatiques essentiels : les précipitations et la température. Ainsi, l'imminence des mesures d'adaptation pour les $61,1 \%$ de la population $\mathrm{du}$ pays qui vivent en milieu rural et dépendent de l'agriculture pour leur subsistance se justifie à plus d'un titre (Emicov, 2007). Aussi, au Bénin, la plupart des écosystèmes des différentes régions agroécologiques sont aujourd'hui marqués par une dégradation du fait de la forte variabilité climatique associée à une plus grande fréquence des phénomènes extrêmes (sécheresse, augmentation des températures, etc.) au cours des trois dernières décennies (Boko, 1988; Afouda, 1990 ; Ogouwalé, 2004, 2006). De même, selon ces mêmes auteurs, un stress thermique supplémentaire conduirait à des sols plus secs et à la réduction des rendements dans les différentes régions agro-écologiques. Les changements climatiques qui se manifestent par des excès de chaleur, des insuffisances de pluies, des excès de pluies, des inondations, des retards de pluies, des sécheresses prolongées, constituent une autre pression encore plus marquante sur l'environnement, réduisant de plus en plus une végétation déjà très perturbée par l'utilisation des techniques de culture inadaptées (Azontondé, 1993). Ces phénomènes ont progressivement réduit le taux de matière organique des sols et leurs niveaux de fertilité. Selon Bokonon-Ganta et al. (2003), à l'horizon 2025, les changements climatiques vont entraîner à l'échelle nationale une baisse considérable des rendements des principales cultures notamment le coton $(-29 \%)$, le riz $(-12 \%)$, le maïs $(-9 \%)$, le niébé (- 5\%), l'igname (-4\%). Ainsi, dans la zone agro-pastorale de production cotonnière du Nord Bénin, notamment à Banikoara, les constats effectués en milieu réel révèlent que les producteurs développent de nombreuses stratégies d'adaptation pour réduire leur vulnérabilité aux changements climatiques. Le changement de cultures, l'amélioration des techniques de gestion des sols font partie de ces mesures (Katé, 2011). C'est pour comprendre les déterminants de l'adaptation aux changements climatiques et formuler des options d'adaptation durables que la présente étude trouve son fondement. Le présent travail vise à évaluer les effets des changements climatiques et des modes de gestion sur la fertilité des sols.

\section{MATERIEL ET METHODES Matériel}

Les matériels utilisés dans le cadre de la réalisation de ce travail sont:01 GPS, 01 boussole, 01 pioche, 01 couteau de pédologue, 01 mètre ruban de $2 \mathrm{~m}$ de long; 01 machette, 01 tarière hollandaise, 01 code Munsell, 01 pelle, le service d'une main d'œuvre, les appareils de laboratoire, et des réactifs.

\section{Localisation de la commune}

La commune de Banikoara est située au Nord-Ouest du Département de l'Alibori, entre $2^{\circ} 05^{\prime}$ et $2^{\circ} 46^{\prime}$ de longitude est et entre $11^{\circ} 02^{\prime}$ et $11^{\circ} 34^{\prime}$ de latitude nord et est limitée au nord par la commune de Karimama, au sud par les communes de Kérou et de Gogounou, à l'Est par la commune de Kandi et à l'Ouest par le Burkina-Faso (Figure 1). Elle couvre une superficie de 4397,2 $\mathrm{km}^{2}$ dont environ 49 $\%$ de terres cultivables et $50 \%$ d'aires 
protégées (Parc National du W du Niger et la zone cynégétique de l'Atacora).

\section{Installation des profils pédologiques}

Six unités pédologiques majeures ont été prises en compte dans l'étude. Dix-huit profils ont été réalisés à raison de 3 par unité de sol. Les profils pédologiques sont des coupes de sol de 2 mètres de long sur 1 mètre de large et 1,50 mètre de profondeur d'orientation Est-Ouest. La face côté soleil est en escalier permettant de descendre dans la fosse tandis que celle du côté opposé mieux éclairée appelée face de description est bien taillée verticalement.

\section{Méthodes}

\section{Démarche suivie dans l'étude}

La réalisation des travaux de terrain pour la collecte des données sur les sols s'est effectuée en deux phases : la première réalisée par Dubroeucq (1971) a duré deux mois (novembre et décembre 1971) et la deuxième exécutée par Katé et al. (2010) a duré un mois $\left(1^{\mathrm{er}}\right.$ au 30 novembre 2010).

Chaque unité a été étudiée dans trois différents arrondissements afin de percevoir les effets des modes de gestion sur le niveau de fertilité des sols tandis que l'impact du temps et des changements climatiques est mesuré à travers la différence de niveau de fertilité entre 1971 et 2010.

La carte des unités de sols (Figure 2) a été réalisée en utilisant les cartes de l'ORSTOM existantes à l'échelle de 1/200.000 (Dubroeucq 1977, Faure 1977 et Viennot 1978).

A l'aide du couteau de pédologue, les différents horizons de chaque profil ont été délimités. Les horizons se distinguent les uns des autres par leur couleur, leur consistance, la nature des constituants minéraux et organiques. Après la description de chaque profil, des échantillons de sols ont été prélevés, étiquetés sur chacun des horizons et expédiés pour leur analyse au laboratoire afin de déterminer les teneurs des différents éléments nutritifs du sol indispensable au bon fonctionnement des plantes et de faire ressortir les déficits éventuels et autres pouvant contraindre leur croissance. Au total, 90 échantillons furent expédiés au Laboratoire des Sciences du Sol, Eau et Environnement (LSSEE) du Centre de Recherche Agricoles d'Agonkamey (CRA-Agonkamey). Les données analytiques ont porté sur: la granulométrie à 5 fractions, le carbone, la matière organique, les cations échangeables, la capacité d'échange cationique (C.E.C ou $\mathrm{T})$, l'azote, le $\mathrm{pH}$, le phosphore assimilable et le taux de saturation en bases.

\section{Méthodes d'analyse des sols}

Les échantillons de sol ont été analysés conformément aux méthodes Tran et Boko (1978) en vigueur au LSSEE. Les échantillons de sol ont été séchés à l'air ambiant puis tamisés à $2 \mathrm{~mm}$ et les refus obtenus ont été pesés et leur pourcentage estimé. Le sol tamisé est utilisé pour l'analyse de la granulométrie, du $\mathrm{pH}, \mathrm{P}_{\mathrm{ass}}$, des cations échangeables et de la capacité d'échange cationique (CEC). Une partie du sol tamisé est broyée à $0,2 \mathrm{~mm}$ pour le dosage de l'azote et du carbone total. Les méthodes suivantes ont été utilisées :

$\checkmark$ la granulométrie par la méthode internationale modifiée par l'emploi de la pipette ROBINSON,

$\checkmark$ la texture à partir du triangle de Durand (1965),

$\checkmark \quad$ le carbone et matière organique par Walkey et Black,

$\checkmark \quad$ l'azote total par la méthode Kjeldahl,

$\checkmark$ pH à l'aide d'un pH-mètre avec $(1 / 2,5)$ comme ratio sol-eau,

$\checkmark$ Phosphore assimilable selon la méthode de BRAY I,

$\checkmark$ Cations échangeables (méthode à l'acétate d'ammonium $\mathrm{pH}=7$ ),

$\checkmark$ le calcium, le magnésium, le potassium et le sodium dosés par spectrophotomètre d'absorption atomique et,

$\checkmark \quad$ la CEC par distillation puis titration.

Méthode d'évaluation des niveaux de fertilité chimique des sols

Le niveau de fertilité des sols a été étudié en utilisant les critères de classification 
définis par SYS (1976). Ainsi, la définition des classes ou sous-classes est basée sur des limitations imposées par les caractéristiques, ainsi que sur le degré d'intensité de ces limitations.

SYS, (1976) a défini cinq degrés d'intensité des limitations : ce sont :

$\checkmark$ 0: Pas de limitation: la caractéristique du sol est optimale ;

$\checkmark \quad 1$ : Limitations légères, se référant à des situations qui pourraient légèrement diminuer les rendements sans cependant imposer des techniques culturales spéciales;

$\checkmark \quad 2$ : Limitations modérées, se référant à des situations qui causent une diminution plus importante des rendements ou la mise en œuvres de techniques culturales spéciales. Ces limitations ne mettent pas la rentabilité en cause ;

3 : Limitations sévères; se référant à des situations qui causent une diminution des rendements ou la mise en œuvre de techniques culturales qui pourraient mettre la rentabilité en cause.

$\checkmark \quad 4$ : Limitations très sévères, se référant à des situations qui ne permettent plus l'utilisation de la terre pour un but précis.

\section{Définition des classes ou niveaux de fertilité}

Les classes ou niveaux de fertilité ont été définies en fonction des limitations en cause et leur degré d'intensité (SYS, 1976). Elles sont les suivantes :

Classe I : Les sols sont de la classe I (niveau de fertilité élevé) lorsque les caractéristiques ne présentent pas ou présentent seulement de faibles limitations.

Classe II : Les sols sont de la classe II (niveau de fertilité moyen) de fertilité lorsque les caractéristiques ne présentent pas plus de trois limitations modérées éventuellement associées à de faibles limitations.

Classe III : Les sols sont de la classe III (niveau de fertilité bas) de fertilité lorsque les caractéristiques présentent plus que trois limitations modérées et associées à une seule limitation sévère.

Classe IV : Les sols sont de la classe IV (niveau de fertilité très bas) de fertilité lorsque les caractéristiques présentent plus d'une limitation sévère.

\section{Analyse statistique des données}

Les teneurs en carbone, en azote, les bases échangeables et capacité d'échange des deux premiers horizons de chacune des six unités pédologiques répétée trois fois ont été soumis à une analyse statistique afin de vérifier l'effet du temps (changement climatique) et l'effet mode d'exploitation (trois répétitions des unités pédologiques) sur les caractéristiques physico-chimiques des sols.

Traitement statistique des données

Les teneurs des différentes caractéristiques physico-chimiques des sols (carbone, azote, base échangeable, Capacité d'Echange Cationique) de chaque unité ont été soumis au test de normalité de Ryan-Joiner et d'homogénéité de variance de Levene. Les analyses ont révélé que les taux de carbone et de l'azote ne sont pas normalement distribués. De ce fait, le test non paramétrique de Kruskal-Wallis a été utilisé pour analyser l'influence du gap de temps (année) et des unités de sol sur le taux de carbone (C) et d'azote $(\mathrm{N})$. Une analyse de la variance à deux facteurs (année et unité de sol) a été effectuée pour analyser l'effet du gap de temps et des unités de sol sur le taux de Base échangeable (BE) et de CEC des sols puisque les données de ces deux variables étaient normalement distribuées et vérifient la condition d'homogénéité des variances. En cas de différence significative d'une variable (BE ou CEC) suivant un facteur (année ou unité de sol), une structuration des moyennes a été réalisée à partir du test de Student Newman Keuls (SNK). Les différentes analyses statistiques ont été réalisées avec le logiciel $\mathrm{R}_{2.15 .3}$ (http:/www.Rproject.org/).

\section{RESULTATS}

Effets des changements climatiques et des modes de gestion sur les caractéristiques morphologiques des sols de 1971 à 2010

Dans la commune de Banikoara, les roches mères et les matériaux originels $(\mathrm{C})$ 
provenant directement de ces roches sont d'origines et de natures variées. On distingue ainsi des matériaux d'origine alluviale et/ou colluviale dont la mise en place est le résultat de transport, et des matériaux d'altération de la roche sous-jacente. Les sols hydromorphes recensés à Kokey, Toura et Founougo sont issus de matériaux d'origine alluviale et/ou colluviale tandis que les autres proviennent de matériaux d'altération de la roche sousjacente.

\section{Variation morphologique des sols hydromorphes de Toura}

Les trois premiers horizons des sols hydromorphes de Toura sont plus rouges (5 YR 4/2) alors que ceux de la profondeur sont gris (10 YR 3/3 et 2,5 Y 5/1) témoignant d'un apport par les eaux de ruissellement des colluvions du village situé à environ $100 \mathrm{~m}$ de la dépression. La description faite de ces sols hydromorphes par Viennot (1970) ne révèle pas la présence des horizons ayant cette couleur rouge brun. Par contre, ce phénomène est absent dans les sols hydromorphes de Founougo et de Kokey situés loin du village.

\section{Variation morphologique des autres unités morphologiques}

Au niveau des autres sols ferrugineux tropicaux de la commune qui proviennent des matériaux d'altération de la roche sousjacente, dans leur état actuel, une modification morphologique absente dans les descriptions de Viennot (1970) a été constatée au niveau de l'horizon de surface.

Effets des changements climatiques et des modes de gestion sur les caractéristiques chimiques des sols de 1971 à 2010

Les Tableaux 2 et 3 montrent qu'en 40 ans (entre 1971 et 2010), les taux de nutriments ont baissé très significativement dans tous les sols quels que soient leurs modes de gestion notamment leurs teneurs en carbone, en azote, en bases échangeables et en capacité d'échange cationique.

Les analyses statistiques faites de ces résultats montrent que le taux de matière organique des différentes unités pédologiques de la Commune de Banikoara a varié très significativement entre 1971 et 2010 (p<0,01). Ce taux de carbone n'a pas varié significativement suivant les unités pédologiques $(H=2,77 ; p>0,05)$. Le taux d'Azote des unités a varié très significativement entre la période de 1971 et 2010 ( $p<0,01)$ mais n'a pas varié suivant les unités pédologiques $(\mathrm{p}>0,05)$.

Les résultats d'analyse de la variance (Tableau 1) indiquent que les taux de bases échangeables (BE) et de capacité d'échange cationique (CEC) ont varié significativement $(p<0,01)$ entre 1971 et 2010 et suivant les unités pédologiques $(\mathrm{p}<0,01)$. Cependant, aucune différence significative $(p>0,05)$ n'a été observée de l'interaction du gap de changements climatiques et des unités pédologiques sur les taux de bases échangeables (BE) et de capacité d'échange cationique des sols (Tableau 2).

Les valeurs moyennes des teneurs en carbone, en azote, en base échangeable et de la Capacité d'Echange Cationique par année et par unité pédologique sont présentées dans le Tableau 2. La comparaison des valeurs moyennes entre année considérant une même unité de sol permet de conclure une diminution du taux de tous les éléments chimique dans le temps quelle que soit l'unité pédologique considérée. 


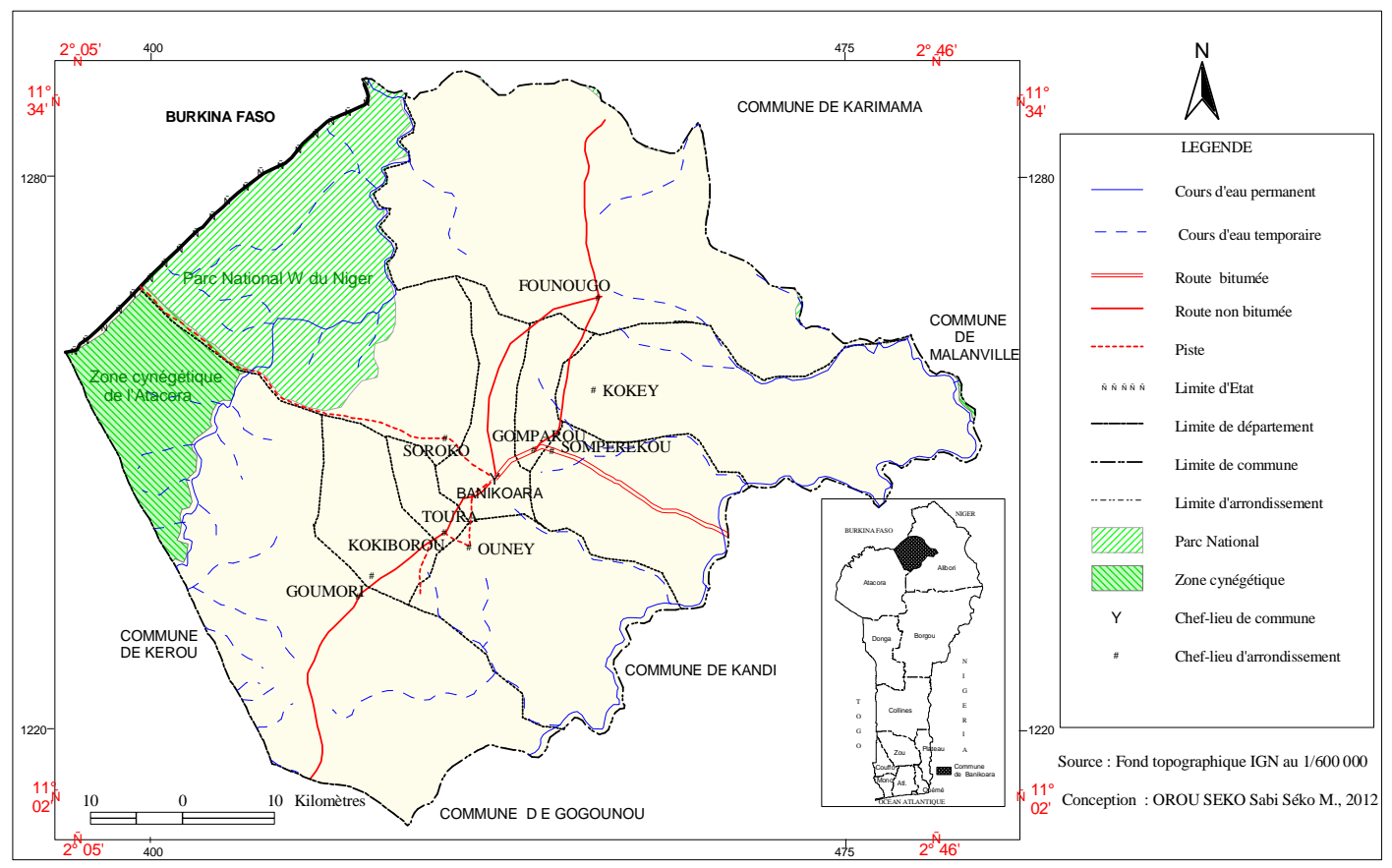

Figure 1 : Carte de localisation de la commune de Banikoara.

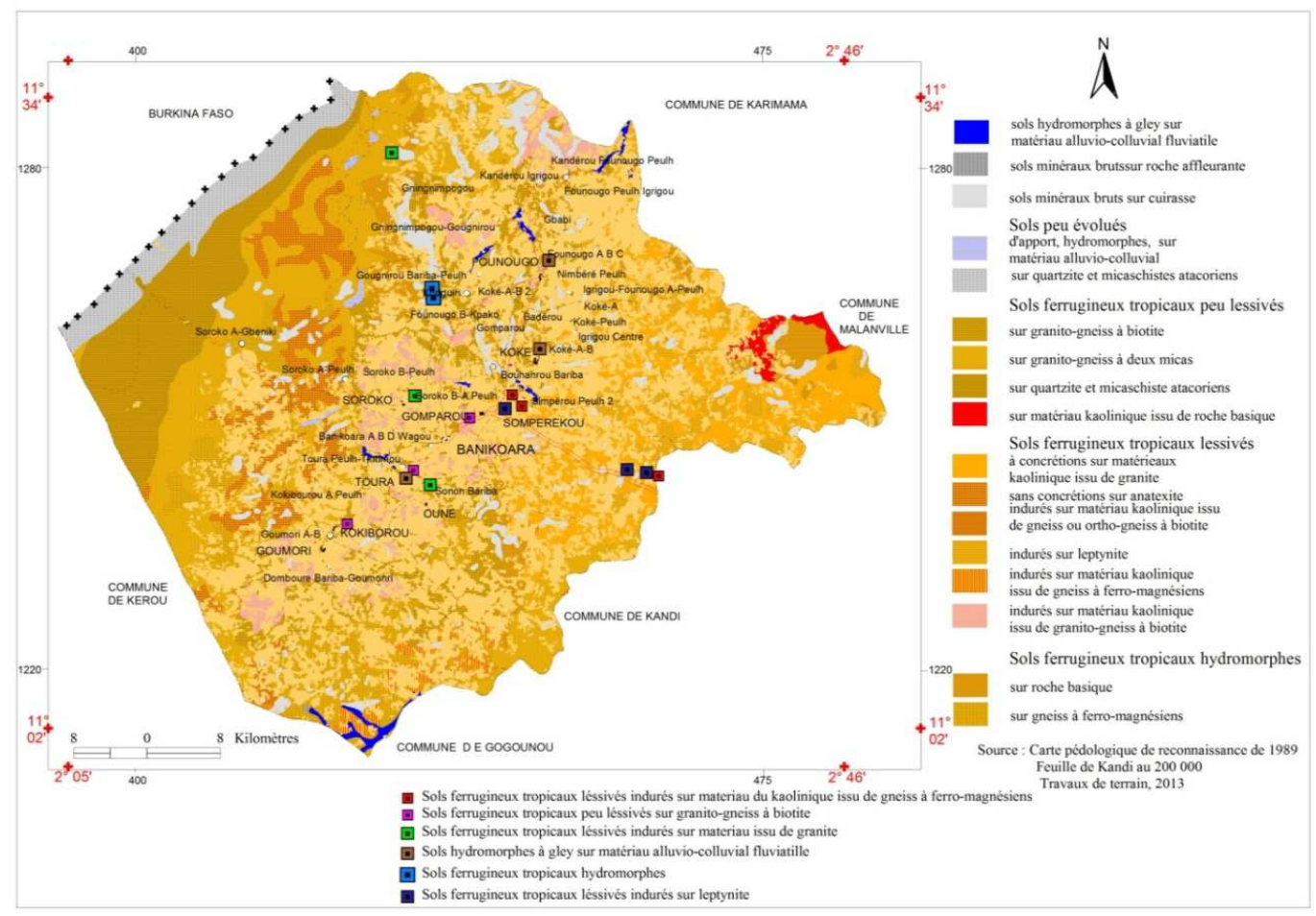

Figure 2 : Carte des sols de la commune de Banikoara. 
Tableau 1 : Critère d'évaluation des classes de limitation des niveaux de fertilité des sols (SYS, 1976).

\begin{tabular}{lcccc}
\hline Limitations & Faibles & Modérées & Sévères & Très \\
\hline $\mathrm{MO}(\%)$ & $>2$ & $1-2$ & $<1$ & $<0,5$ \\
$\mathrm{~N}(\%)$ à $\mathrm{pH} 6$ & $>0,08$ & $0,045-0.08$ & $<0,045$ & $<0,03$ \\
$\mathrm{P}$. ass $(\mathrm{mg} / \mathrm{kg})$ & $>20$ & $10-20$ & $<10$ & $<5$ \\
$\mathrm{~K}(\mathrm{Cmol} / \mathrm{kg})$ & $>0,4$ & $0,2-0,4$ & $<0,2$ & $<0,1$ \\
Somme des cations $(\mathrm{Cmol} / \mathrm{kg})$ & $>10$ & $5-10$ & $<5$ & $<2$ \\
Saturation en base $(\mathrm{Cmol} / \mathrm{kg})$ & $>60$ & $40-60$ & $<40$ & $<15$ \\
C.E.C $(\mathrm{Cmol} / \mathrm{kg})$ & $>25$ & $10-25$ & $<10$ & $<5$ \\
\hline
\end{tabular}

Tableau 2 : Effet des changements climatiques et unités pédologiques sur les bases échangeables (BE) et la capacité d'échange cationique (CEC) des sols.

\begin{tabular}{llllll}
\hline \multirow{2}{*}{ Source de variation } & \multicolumn{3}{c}{ Base échangeable } & \multicolumn{2}{c}{ Capacité d'échange cationique } \\
\cline { 2 - 6 } & DDL & SCE & F & SCE & F \\
\hline Année & 5 & 500,9 & $11,17^{* * *}$ & 860,1 & $10,51^{* * *}$ \\
Unité & 1 & 359,2 & $40,06^{* * *}$ & 362,7 & $22,17^{* * *}$ \\
Année*Unité & 5 & 61,4 & 1,37 & 66,9 & 0,81 \\
\hline
\end{tabular}

Tableau 3 : Teneur moyenne des éléments chimiques des unités pédologiques en 1971 et 2010.

\begin{tabular}{|c|c|c|c|c|c|c|c|c|c|}
\hline \multirow{2}{*}{ Unité } & \multirow{2}{*}{ Année } & \multicolumn{2}{|c|}{$\mathrm{C}$} & \multicolumn{2}{|c|}{$\mathbf{N}$} & \multicolumn{2}{|c|}{$\mathbf{B E}$} & \multicolumn{2}{|c|}{ CEC } \\
\hline & & $\mathrm{m}$ & se & $\mathbf{m}$ & se & $\mathbf{m}$ & se & $\mathbf{m}$ & se \\
\hline & 1970 & 2,51 & 0,16 & $\mathbf{0 , 2 0}$ & 0,02 & 9,71 & 1,73 & 11,51 & 2,34 \\
\hline \multirow[t]{2}{*}{ A } & 2010 & 0,49 & 0,16 & 0,09 & 0,02 & 3,66 & 1,73 & 6,48 & 2,34 \\
\hline & 1971 & 2,34 & 0,16 & 0,19 & 0,02 & $9, \mathbf{3 0}$ & 1,73 & 12,25 & 2,34 \\
\hline \multirow[t]{2}{*}{ B } & 2010 & 0,57 & 0,16 & 0,06 & 0,02 & 3,42 & 1,73 & 7,07 & 2,34 \\
\hline & 1971 & 2,19 & 0,16 & 0,19 & 0,02 & 12,01 & 1,73 & 16,08 & 2,34 \\
\hline \multirow[t]{2}{*}{$\mathrm{C}$} & 2010 & 0,43 & 0,16 & 0,04 & 0,02 & 2,06 & 1,73 & 5,17 & 2,34 \\
\hline & 1971 & 2,25 & 0,16 & 0,19 & 0,02 & 17,52 & 1,73 & 22,83 & 2,34 \\
\hline \multirow[t]{2}{*}{ D } & 2010 & 1,55 & 0,16 & 0,12 & 0,02 & 13,03 & 1,73 & 17,84 & 2,34 \\
\hline & 1971 & 2,60 & 0,16 & $\mathbf{0 , 2 2}$ & 0,02 & 15,86 & 1,73 & 22,40 & 2,34 \\
\hline \multirow[t]{2}{*}{$\mathbf{E}$} & 2010 & 0,72 & 0,16 & 0,07 & 0,02 & 13,52 & 1,73 & 19,46 & 2,34 \\
\hline & 1971 & 2,61 & 0,16 & 0,21 & 0,02 & 13,78 & 1,73 & 18,61 & 2,34 \\
\hline $\mathbf{F}$ & 2010 & 0,60 & 0,16 & 0,06 & 0,02 & 4,58 & 1,73 & 9,57 & 2,34 \\
\hline
\end{tabular}


S. KATE et al. / Int. J. Biol. Chem. Sci. 10(1): 120-133, 2016

Tableau 4 : Niveaux de fertilité des sols sur une profondeur de 0-20 cm en 1970.

\begin{tabular}{|c|c|c|c|c|c|c|c|c|c|c|c|c|}
\hline Caractéristiques & 1 & Limitation & 2 & Limitation & 3 & Limitation & 4 & Limitation & 5 & Limitation & 6 & Limitation \\
\hline $\mathrm{MO}(\%)$ & 4,32 & 1 & 4,03 & 1 & 3,77 & 1 & 3,87 & 1 & 4,47 & 1 & 4,49 & 1 \\
\hline $\mathrm{N}(\%)$ à $\mathrm{pH} 6$ & 0,20 & 1 & 0,19 & 1 & 0,19 & 1 & 0,19 & 1 & 0,22 & 1 & 0,20 & 1 \\
\hline P.ass $(\mathrm{Mg} / \mathrm{kg})$ & 77 & 1 & 82 & 1 & 133 & 1 & 93 & 1 & 91 & 1 & 40 & 1 \\
\hline $\mathrm{K}(\mathrm{Cmol} / \mathrm{kg})$ & 0,17 & 3 & 0,12 & 3 & 0,16 & 3 & 0,33 & 2 & 0,14 & 3 & 0,15 & 3 \\
\hline Somme des Cations & 9,71 & 2 & 9,29 & 2 & 12 & 1 & 17,51 & 1 & 15,86 & 1 & 13,77 & 1 \\
\hline Saturation en base $\%$ & 83 & 1 & 75 & 1 & 76 & 1 & 82 & 1 & 71 & 1 & 65 & 1 \\
\hline C.E.C (Cmol/kg) & 11,50 & 2 & 12,25 & 2 & 16,08 & 2 & 22,82 & 2 & 22,4 & 2 & 18,61 & 2 \\
\hline Classe de fertilité & - & II & - & II & - & II & - & II & - & II & - & II \\
\hline Niveau de fertilité & & Moyen & & moyen & & moyen & & moyen & & moyen & & moyen \\
\hline
\end{tabular}

Tableau 5 : Niveaux de fertilité des sols sur une profondeur de 0-20 cm en 2013.

\begin{tabular}{|c|c|c|c|c|c|c|c|c|c|c|c|c|}
\hline Caractéristiques & 1 & Limitation & 2 & Limitation & 3 & Limitation & 4 & Limitation & 5 & Limitation & 6 & Limitation \\
\hline $\mathrm{MO}(\%)$ & 0,83 & 3 & 0,98 & 1 & 0,73 & 1 & 2,67 & 1 & 1,24 & 2 & 0,97 & 1 \\
\hline $\mathrm{N}(\%)$ à $\mathrm{pH} 6$ & 0,08 & 2 & 0,05 & 2 & 0,04 & 2 & 0,12 & 1 & 0,20 & 1 & 0,06 & 2 \\
\hline P.ass $(\mathrm{Mg} / \mathrm{kg})$ & 50,66 & 1 & 2,33 & 4 & 3,33 & 4 & 22 & 1 & 25 & 1 & 2 & 4 \\
\hline $\mathrm{K}(\mathrm{Cmol} / \mathrm{kg})$ & 0,24 & 2 & 0,16 & 3 & 0,16 & 3 & 0,3 & 2 & 0,15 & 3 & 0,18 & 3 \\
\hline Somme des Cations & 3,65 & 3 & 3,41 & 3 & 2,05 & 3 & 13,03 & 1 & 13,52 & 1 & 4,58 & 3 \\
\hline Saturation en base $\%$ & 93 & 1 & 54 & 2 & 42 & 2 & 76 & 1 & 60 & 2 & 40 & 2 \\
\hline C.E.C (Cmol/kg) & 6,48 & 3 & 7,06 & 3 & 5,17 & 3 & 17,84 & 2 & 19,45 & 2 & 9,55 & 3 \\
\hline Classe de fertilité & - & IV & - & IV & - & IV & - & II & - & II-III & - & IV \\
\hline Niveau de fertilité & & Très bas & & Très bas & & Très bas & & moyen & & moyen à bas & & Très bas \\
\hline
\end{tabular}




\section{DISCUSSION}

Variation morphologique des sols hydromorphes de Toura

Une variation morphologique des sols hydromorphes de Toura a été observée. Elle peut être due aussi bien à la dégradation du couvert végétal dont la force de filtration du ruissellement est plus faible qu'à l'extension $\mathrm{du}$ village. L'effet dégradant des changements climatiques sur la végétation a été décrit par Vissin (2007) qui a montré que les changements climatiques ont entraîné la dégradation $\mathrm{du}$ couvert végétal, la modification des états de surface et les écoulements du bassin béninois du fleuve Niger. Casenave et Valentin (1988) ont déjà constaté que la réduction de la végétation modifie les états de surface et augmente le ruissellement vers le bas versant. C'est donc la position topographique qui favorise les modifications morphologiques induites par la dégradation de la végétation due aux changements climatiques et aux modes de gestion des sols.

Effets des changements climatiques et des modes de gestion sur les caractéristiques chimiques des sols de 1971 à 2010

Leurs caractéristiques chimiques des sols sont comparables à celles des sols ferrallitiques dégradés décrits par Poss (1991) ; Azontondé (1993) et Lal (1997)

Une interaction du gap de changements climatiques et des unités pédologiques sur les taux de bases échangeables et de capacité d'échange cationique des sols a été observée et traduit que l'effet changements climatiques dans le temps sur les bases échangeables (BE) et sur la capacité d'échange cationique des sols ne dépendent pas des unités pédologiques et vice versa (Tableau 3). De ces résultats, il ressort que la grande différence constatée entre les caractéristiques chimiques des sols de 1971 à 2010 est moins imputable aux modes de gestion qu'aux perturbations (changements) climatiques.

Effets des changements climatiques et des modes de gestion sur les niveaux de fertilité et de dégradation des sols

Les changements climatiques ont induit de 1971 à 2010 une importante et significative diminution des teneurs en éléments chimiques et des niveaux de fertilité dans tous les sols. Cette baisse peut être due aux effets que les changements climatiques ont eus sur la diminution drastique des taux de matière organique et d'azote du sol. En effet, l'augmentation de la fréquence et de la durée des poches de sécheresse peut réduire la croissance et la biomasse végétative. Aholoukpè (2010) a expliqué cette baisse drastique des taux de nutriments dans le département du Plateau dans le Sud-Bénin par l'absence de fertilisation minérale et l'exportation totale ou partielle par les résidus de récolte. Bacyé (1993) avait déjà constaté que les systèmes de culture avaient une influence importante sur l'évolution du statut organique et minéral des sols ferrugineux et hydromorphes de la zone soudano-sahélienne de la "Province du Yatenga au Burkina Faso. Azontondé (1993) a obtenu les mêmes résultats sur les sols ferrallitiques dans le Sud-Bénin. Ces diminutions importantes des teneurs en éléments chimiques en 40 ans ont induit une forte baisse des niveaux de fertilité des sols. Ainsi, tous les sols de la commune qui avaient un niveau de fertilité moyen en 1971 (Tableau 4) sont passés actuellement à un niveau de fertilité très bas (Tableau 5). En dehors du sol hydromorphes dont le niveau de fertilité est resté moyen et des sols ferrugineux tropicaux hydromorphes sur roche basique dont le niveau de fertilité se situe entre moyen et bas. C'est dire qu'aucun des modes de gestion utilisés par les producteurs, n'a pu relever ou conserver la 
fertilité des sols. Si les sols hydromorphes et les sols ferrugineux hydromorphes sur roche basique sont à des niveaux de fertilité respectivement moyens et situés entre moyens et bas, c'est dû pour les premiers à leur situation de bas de pente et pour les seconds à leur matériau originel déjà riche en cations basiques $\left(\mathrm{Ca}^{++}\right.$et $\left.\mathrm{Mg}^{++}\right)$. Les sols hydromorphes s'enrichissent chaque année en éléments fins d'origine organo-minéral (Dabin, 1956) tandis que les ferrugineux hydromorphes renouvellent leurs stocks à partir de la roche mère. Par contre, les autres sols de la commune qui sont des sols ferrugineux situés à un niveau topographique plus élevé (exondés) subissent du fait des fréquentes poches de sécheresse, des retards de pluies, des insuffisances de pluies annuelles et les mauvaises répartitions des pluies, une diminution du couvert végétal, une accélération des phénomènes d'érosion et une dégradation morphologique, physicochimique et biologique (Roose, 1981; Lal, 1997 ; Feller et Beare, 1997). Il se pose alors la question de savoir si les modes de gestion des sols à Banikoara ne sont pas tous comparables aux systèmes traditionnels dégradant de culture sur brûlis ou au système de culture sans restitution ou à restitution partielle pratiqué sur les plateaux du SudBénin (Azontondé, 2000) ou plus précisément dans le département du Plateau au Sud-Bénin (Aholoukpè, 2013). En effet, à la récolte des céréales, par leur premier pâturage, les bovins rabattent les tiges qui sont par la suite broutées au deuxième. Lors du troisième passage, le sol est débarrassé de toute sa matière organique et il ne reste que des brindilles. De plus, pendant la préparation des sols pour la culture suivante, les crottes des bovins et les tiges de cotonniers sont brulées. Aussi, dans cette localité, les tiges de céréales sont exportées par les producteurs et servent à la confection des palissades, la fabrication de la potasse traditionnelle utilisée pour ramollir la sauce de gombo, ou bien la fabrication du savon traditionnel. Ces modes de gestion de la matière organique, pratiqués annuellement dans la commune de Banikoara contribuent à une baisse croissante du stock organique du sol et à l'accélération de sa dégradation. Ils pourraient expliquer aussi l'épuisement des sols en nutriments constatés par Van der pool et al. (1993) ; Boko et al. (1997). On peut retenir que la situation actuelle de la gestion de la fertilité des sols n'est pas satisfaisante. Les nouvelles technologies introduites par la vulgarisation sont peu adoptées, car leurs conditions de réalisation ne sont pas remplies; l'objectif du producteur étant l'extension des superficies cultivées afin d'accroître la production du coton. Il convient alors de proposer des méthodes adéquates dans le but de relever puis de maintenir la fertilité des sols dans la commune de Banikoara si l'on veut éviter une grave crise alimentaire les années à venir. L'intensification agricole couplée au système amélioré de production intégrant l'assolement-rotation, l'application des engrais pour chaque culture, la compartimentation des blocs de culture à l'aide de cactus pour protéger les parcelles contre l'érosion et la dégradation du sol par les bovins transhumants, la gestion in situ de la matière organique l'usage des mesures anti érosives, etc. peuvent être recommandées dans cette localité.

\section{Conclusion}

L'étude des effets des changements climatiques et des modes de gestion sur la fertilité des sols de la commune de Banikoara a permis de comparer les paramètres de fertilité des sols tels que les caractéristiques morphologiques, physiques et chimiques de toutes les unités pédologiques de la commune de 1971 à ceux de 2010 et a fait ressortir : iDes modifications dans la morphologie des 
horizons de surface des sols par recouvrement des horizons superficiels des sols hydromorphes et par décapage et formation de pellicules de battance sur les horizons de surface des autres sols rouges ferrugineux tropicaux; ii- Une importante et significative diminution des teneurs en éléments chimiques dans tous les sols étudiés; iii- Une baisse importante des niveaux de fertilité des sols qui sont passés de moyen en 1971 à très bas en 2010 en dehors des sols hydromorphes et des sols ferrugineux hydromorphes sur roche basique. La position topographique basse a favorisé la conservation des caractéristiques chimiques de ces deux sols. iv- Les modes de gestion n'ont pas montré un effet significatif sur la baisse des caractéristiques des sols d'une unité pédologique à l'autre, ce qui prouve que la dégradation des sols en 40 ans est due plus au temps et au climat autrement dit aux changements climatiques qu'aux modes de gestion des sols.

\section{REFERENCES}

Adam KS, Boko K. 1993. Le Bénin. Ed du Flamboyant, EDICEF, Cotonou, Vanves.

Afouda F., 1990. L'eau et les cultures dans le Bénin central et septentrional : Etude de la variabilité des bilans de l'eau dans leurs relations avec le milieu rural de la savane africaine. Thèse de Doctorat nouveau régime: Univ. Paris IV (Sorbone), Institut de Géographie, 428 p.

Aholoukpè NSH. 2013. Matière organique du sol et développement du palmier à huile sous différents modes de gestion des feuilles d'élagage. Cas des palmeraies villageoises du département du Plateau au Bénin. Thèse de doctorat unique soutenue à l'Université d'AbomeyCalavi, 273 p.

Azontondé HA. 1993. Dégradation et restauration des terres de barre (sols ferralitiques faiblement désaturés argilo- sableux) au Bénin. La gestion conservatoire de l'eau, de la biomasse et de la fertilité des sols (GCES). Cah. ORSTOM, sér. Pédol., 2 : 217-226.

Azontondé HA. 2000. Dynamique de la matière organique et de l'azote dans le système Mucuna-maïs sur un sol ferrallitique (terres de barre) au SudBénin. Thèse de doctorat unique soutenue à l'Université de Montpellier. ORSTOM, $241 \mathrm{p}$.

Bacyé R. 1993. Influence des systèmes de culture sur l'évolution du statut organique et minéral des sols ferrugineux et hydromorphes de la zone soudano-sahélienne (Province du Yatenga, Burkina Faso). Document ORSTOM-Montpellier, n6, $243 \mathrm{p}$.

Baldock A, Kay BD. 1987. Influence of cropping history and chemical treatments on the water-stable aggregation of a silt loam soil. Cano J. Soil. Sei., 67: 501511.

Banque Mondiale. 2006. Développement durable dans une dynamique de transformation des institutions, croissance et qualité de vie, $86 \mathrm{p}$.

Boko KA, Kpagbin AG. 1997. Bilan des Eléments Nutritifs dans les Systèmes de Production au Nord-Bénin : Cas de Banikoara. INRAB/CENAP: Cotonou.

Boko M. 1988. Climats et communautés rurales du Bénin: Rythmes climatiques et rythmes de développement. Thèse de Doctorat d'Etat ès Lettres et Sciences Humaines : Univ. de Bourgogne, Dijon, 2 volumes, $601 \mathrm{p}$.

Bokonon-Ganta AH, Bernal JS, Pietrantonio PV, Setamou M. 2003. Survivorship and development of fall armyworm, Spodoptera frugiperda (J.E. Smith) (Lep., Noctuidae) on conventional and transgenic maize cultivars expressing Baccilus thuringiensis Cry 9C and Cry 
1A (b) endotoxins. International Journal of Pest Management, 49: 169-175.

Casenave A, Valentin C. 1988. Les Etats de Surface de la Zone Sahélienne. ORSTOM: Paris ; 202.

Combeau A. 1960. Quelques Facteurs de la Variation de l'Indice Structurale dans Certains Sols Ferrallitiques. C. R. Académ. Agric.: France; 9-115.

Conway G, Toenniessen G. 2003. Science for African Food Security. Science, New Series, 299: 1187-1188.

Dabin B. 1956. Contribution à l'étude de la fertilité des terres de barre, Agron. Trop., 11(4): 490-506.

Dubroeucq D. 1977. Carte pédologique de reconnaissance de la République Populaire du Bénin à 1/200.000. Notice explicative des Feuille de Parakou. ORSTOM, Paris, 37 p.

Dutartre P. 1993. Horizons de surface de sols ferrugineux tropicaux cultivés d'Afrique de l'Ouest (Burkina Faso et Mali). Etude des microstructures par l'approche fractale. Thèse, Univ. Nancy 1, 153 p.

FAO. 2007. L'adaptation aux changements climatiques centrée sur les personnes: intégration des questions de parité. Rome, Italie.

Faure P. 1977. Carte pédologique de reconnaissance de la République Populaire du Bénin à 1/200.000. Notice explicative des Feuilles de Natitingou et Porga. ORSTOM, Paris, 67 p.

Feller C, Beare MH. 1997. Physical control of soil organic matter dynamics in the tropics. Geoderma, 79: 69-116.

GIEC. 2007. Bilan des changements climatiques. Contribution des Groupes de travail I, II et III au quatrième Rapport d'évaluation du Groupe d'experts intergouvernemental sur l'évolution du climat [Équipe de rédaction principale, Pachauri, R.K. et Reisinger, A.]. GIEC. Genève : 103 p.
Gijsman AJ, Thomas RJ. 1995. Aggregate size distribution and stability of an oxisolunder legumes-based and pure grass pastures in eastem Columbian savannas. Austr. Soi. Res., 33: 153-165.

IPCC (Intergovernmental Panel on Climate Change). 1996. Climate Change 1995. The Science of Climate Change. Contribution of Group I to the second Assessment Report of the IPCC. Press. Caveli, California; $572 \mathrm{p}$.

Issa. 1995. Impacts potentiels d'un changement climatique dû au doublement du $\mathrm{CO}_{2}$ atmosphérique sur l'agriculture en République du Bénin. Mémoire de DESS. Université Senghor d'Alexandrie, $113 \mathrm{p}$.

Katé. 2011. Manifestations des changements climatiques et perceptions des producteurs dans les zones cotonnières : cas de l'arrondissement de Founougo (commune de Banikoara). Mémoire de DEA. Université d'Abomey-Calavi, 65 p.

Lal R. 1997. Long-term tillage and maze monoculture effects on a tropical alfisol in Western Nigeria - I Crop yield and soil physical properties. Land Degradation Rehabil, 42: 145-160.

Ogouwalé E. 2004. Changements climatiques et sécurité alimentaire dans le Bénin méridional. Mémoire de DEA, UAC/EDP/FLASH, 119 p.

Ogouwalé E. 2006. Changement climatique dans le bénin méridionale et central : Indicateurs, scénarios et perspectives de la sécurité alimentaire. Thèse de Doctorat de Géographie: Université d'Abomey-Calavi, Dynamique des systèmes climatiques, $248 \mathrm{p}$.

OMM, PNUE. 2002. Rapport mondial sur le développement humain, $86 \mathrm{p}$.

OMM. 2009. Rapport général des Séminaires Itinérants pour les Paysans sur le Temps et le Climat en Afrique de l'Ouest [en 
ligne]. Ouagadougou: Mars 2009. (consulté le 18.12.2013).

Roose E. 1981. Dynamique actuelle des sols ferrallitiques et ferrugineux tropicaux d'Afrique Occidentale. Travaux et documents de l'ORSTOM. $\mathrm{N}^{\circ}$ 130, 566 p.

SRPA (Stratégie de Relance de la Production Agricole). 2008. Plan d'action d'urgence, période 2008-2010, Togo, 72 p.

Tran Vin A, Boko A. 1978. Procédures d'Analyses Physiques et Chimiques des Sols, Eaux et Végétaux. CENAP/INRAB: Agonkanmey, 251 p.

Valentin C. 1981. Organisation pelliculaire superficielle de quelques sols de région subdésertique

(Agadès-Niger).

Dynamique et conséquences sur l'économie en eau : Thèse de $3^{\text {ème }}$ cycle,
Univ. Paris VII. Etudes et Thèses, ORTSTOM, 1985, 259 p.

Van der Pool F, Gogan AC, Dagbénonbakin G. 1993. L'épuisement des sols et sa valeur économique dans le département du Mono, Bénin. DRA/RAMR. 79 p. KIT, Amsterdam.

Viennot M. 1978. Carte pédologique de reconnaissance de la République Populaire du Bénin à 1/200.000. Notice explicative de la feuille de KandiKarimama. ORSTOM Paris, 47 p.

Vissin WE. 2007. Impact de la vulnérabilité climatique et de la dynamique des états de surface sur les écoulements du bassin béninois du fleuve Niger. Thèse de Doctorat unique, Dijon-France, 285p. 